\title{
The Correlation of Health Literacy with Quality of Life in Asthmatic Patients
}

\author{
Mina Kazemi ${ }^{1}$, Maasoumeh Barkhordari-Sharifabad ${ }^{2}$, Khadijeh Nasiriani ${ }^{3}$ \\ Hossein Fallahzadeh ${ }^{4}$
}

\begin{abstract}
Background \& Aims: Health literacy is defined as the degree to which an individual can obtain, communicate, process, and understand basic health information and services to make appropriate health decisions. Asthma is a common, chronic disease, which has a deep impact on life and physical, social, and mental performance, group activities, and even the economic advancement of patients, all of which are factors that affect their quality of life. On the other hand, effective management of the disease requires identifying effective factors, including health literacy. Therefore, with regard to the importance of asthma and quality of life of those with this condition, and given the effect of health literacy on this issue, the present study aimed to determine the relationship between health literacy and quality of life of asthmatic patients referring to healthcare clinics of Shahid Sadoughi University of Medical Sciences, Yazd, Iran, in 2018.

Materials \& Methods: This correlational and descriptive study was performed on 300 asthmatic patients selected by simple random sampling. The inclusion criteria were the age range of 18-60 years, Farsi language proficiency, being literate, lack of malignancy and other underlying diseases, and lack of physical and psychological disabilities. On the other hand, the exclusion criteria were living in other provinces and lack of referring to the healthcare centers for several months. Data were collected using demographic characteristics questionnaire (age, gender, marital status, level of education, and occupational status), patient-reported asthma life quality questionnaire, which included 20 items in four physical, psychological, social, and health concern aspects, and patient-reported test of functional health literacy in adults (TOFHLA). Data analysis was performed in SPSS version 16 using descriptive and inferential statistics, including mean and standard deviation, absolute and relative frequency, and Kolmogorov-Smirnov test (to evaluate the normal distribution of the data). In case of a lack of normal distribution $(\mathrm{P}<0.05)$, we applied Spearman's test to determine the relationship between the variables.

Results: In this study, most patients were female (52.3\%) and married (87.3\%) with the mean age of $43.9 \pm 11.08$ years and minimum and maximum ages of 19 and 60 years, respectively. In addition, most of the subjects $(73.3 \%)$ used the option of asking the physician and health care staff to receive information about asthma. The mean health literacy score of the subjects was $100.78 \pm 28.55$ and the highest and lowest scores were related to the components of evaluation (68.5) and access (55.36), respectively. In addition, the majority of the participants (45\%) had inadequate health literacy. Moreover, the mean life quality score of the participants was $46.91 \pm 5.32$, and the lowest and highest mean scores were related to the physician $(10.66 \pm 2.88)$ and health concern $(17.41 \pm 2.88)$ dimensions, respectively. According to the results, there was no significant difference between the variables of health literacy and life quality with gender $(\mathrm{P}>0.05)$. However, there was a significant relationship between health literacy and marital status, meaning that single individuals had higher health literacy, compared to married subjects $(\mathrm{P}<0.001)$. However, there was no significant relationship between marital status and life quality $(\mathrm{P}=0.61)$. Kruskal-Wallis test results were indicative of a significant relationship between health literacy with the level of education and occupational status $(\mathrm{P}<0.001)$. In other words, there was a significant difference between those with MSc and BSc degrees and those with associate and below diploma degrees $\mathrm{P}<0.001$ ). In addition, student and employed patients had a significantly different life quality, compared to housewives and retired participants $(\mathrm{P}<0.001)$. However, there was no significant difference between the level of education and occupational status regarding the quality of life $(\mathrm{P}>0.05)$. Spearman's test results demonstrated a significant, reverse association between age and health literacy $\mathrm{Rho}=-0.76, \mathrm{P}<0.001)$, which was not observed between age and life quality $(\mathrm{Rho}=-0.01, \mathrm{P}=0.82)$. Moreover, no statistically significant relationship was found between health literacy and its components with quality of life and its dimensions $(\mathrm{P}>0.05)$.
\end{abstract}

\footnotetext{
1. MS of Medical-Surgical Nursing, College of Medical Science, Islamic Azad University, Yazd Branch, Yazd, Iran 2. Assistant Professor, Department of Nursing, College of Medical Science, Islamic Azad University, Yazd Branch, Yazd, Iran (Corresponding author) Tel: 00983538210540-41 Email: Barkhordari_m@iauyazd.ac.ir

${ }^{3}$. Associate Professor, Department of Nursing, Nursing and Midwifery Research Center, Mother \& Newborn Health Research Center, Shahid Sadoughi University of Medical Sciences, Yazd, Iran

${ }^{4}$. Professor, Department of Biostatistics and Epidemiology, School of Health, Shahid Sadoughi University of Medical Sciences, Yazd, Iran
} 
Conclusion: According to the results of the study, the mean health literacy score of the subjects was 100.78; therefore, the overall health literacy of the subjects was below moderate. In addition, according to the ranking, the health literacy of most of the subjects was inadequate. This indicates the need to use effective strategies to improve the health literacy of asthmatic patients because adequate health literacy is essential for patient participation in health care systems and making appropriate health decisions and leads to the empowerment of people to use relevant information and instructions. It seems that due to insufficient health literacy in the present study, it should be considered by health care workers. The mean life quality score of the patients was $46.91 \pm 5.32$, which showed the below moderate life quality of the participants. Similar to other diseases, asthma affects the lives of patients in many ways, in a way that it becomes the main concern of patients. This condition extremely decreases the quality of life of individuals. Therefore, it is not uncommon for people with asthma to have poor living standards. Nevertheless, it is necessary to pay more attention to the quality of life of these patients and use and implement appropriate interventions to improve their quality of life. According to the results, the level of health literacy and quality of life of patients with asthma was not favorable and there was no significant relationship between health literacy and its components with quality of life and its dimensions. Therefore, it is suggested that more studies be performed to evaluate the intermediating factors that could affect these variables to better understand this condition. Moreover, it is possible to develop health literacy skills and improve the quality of life of asthmatics by developing comprehensive programs, creating simple and understandable educational materials, and effective psychological interventions. The lack of a relationship between health literacy and quality of life in this study does not mean that these variables have no effect on each other. In fact, the relationship might be indirect, and a better understanding is required to assess other intermediating simultaneously with these two variables. It is recommended that variables such as adherence to treatment be assessed in future studies as an intermediary variable between health literacy and life quality of patients with asthma.

Keywords: Health Literacy, Life Quality, Asthma

\section{Conflict of Interest: No}

How to Cite: Kazemi M, Barkhordari-Sharifabad M, Nasiriani KH, Fallahzadeh H. The Correlation of Health Literacy with Quality of Life in Asthmatic Patients. Iran Journal of Nursing. 2019; 32(121):54-67.

Received: 14 Sep 2019

Accepted: 14 Dec 2019 


\title{
ارتباط سواد سلامت با كيفيت زندكى در بيماران مبتلا به آسم
}

\author{
مينا كاظمى'، معصومه برخور دارى شريف آبادَ، خديجه نصيريانى'، حسين فلاح زادهء
}

\section{جكيده}

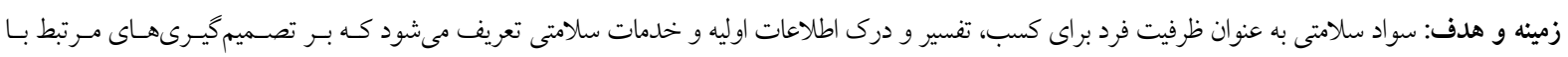

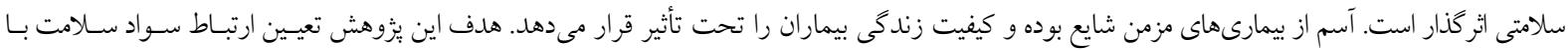

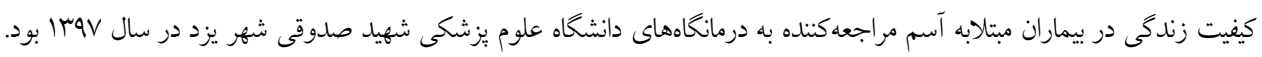

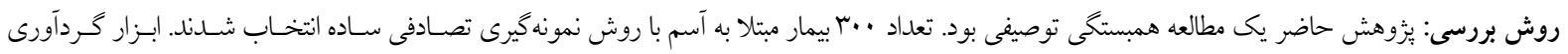

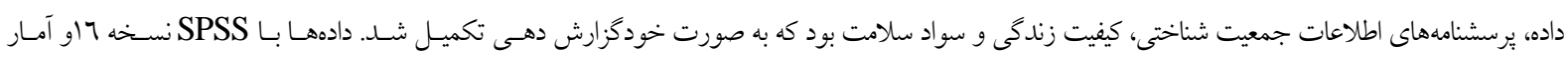
توصيفى و استنبطى تحليل شدند.

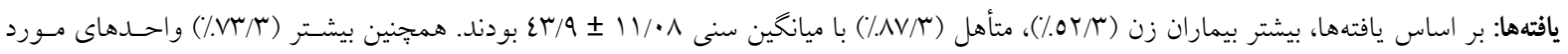

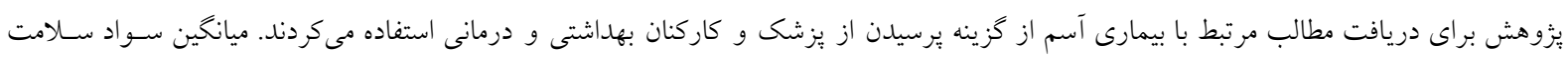

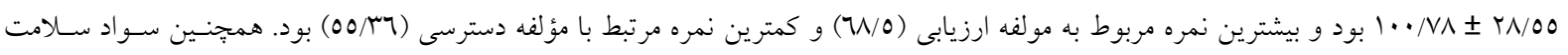

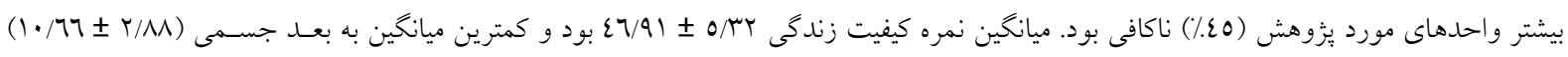

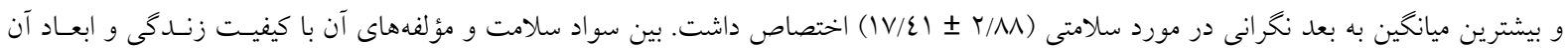

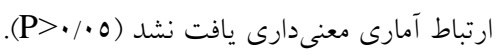

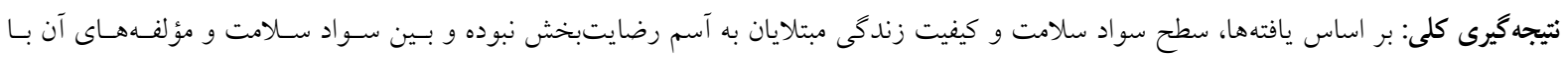

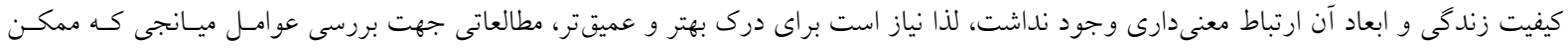

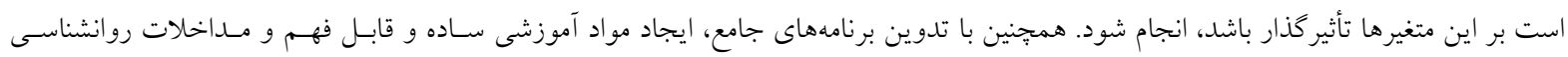

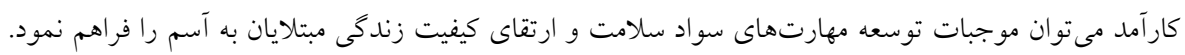
كليد وازهها: سواد سلامت، كيفيت زندگى، آسم

شماره تماس:

$$
\text { (نويسنده مسئول) }
$$

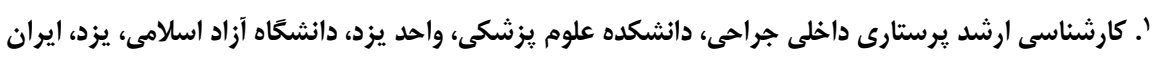

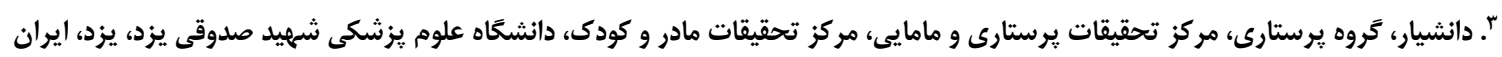

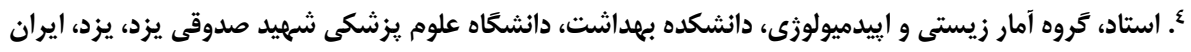



ترويج و حفظ سلامت خوب را در بر مى گيرد (·cr) سواد

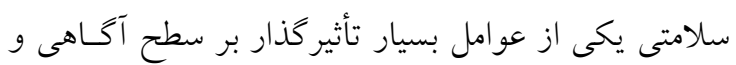

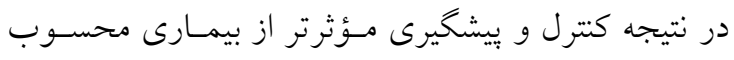

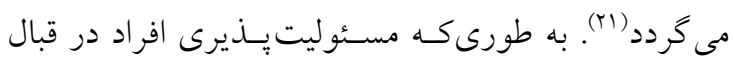

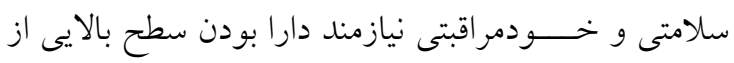

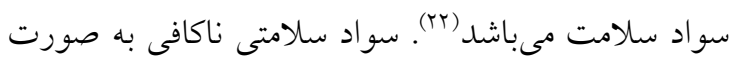
توانايى محدود افراد براى كسب تفسير و درك اطلاعـات اوليه خدمات سلامتى براى تصميم گيرى مناسب در حيطه

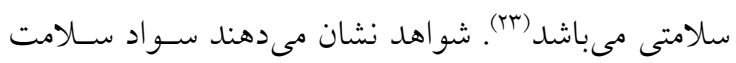
پايين با نتايج نامطلوب بهلهاشتى، مراجعه مكرر به يزشك،

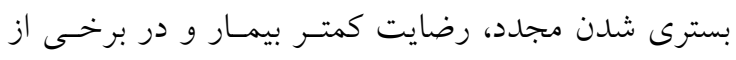

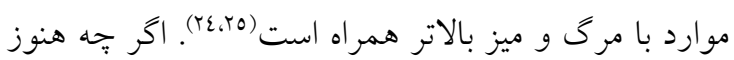

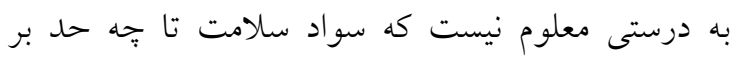

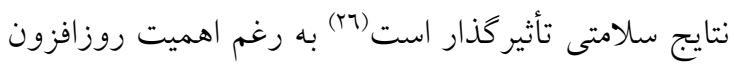

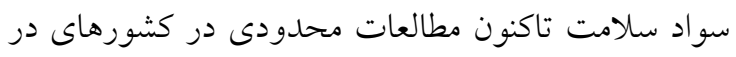

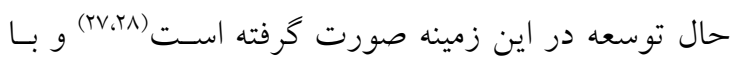

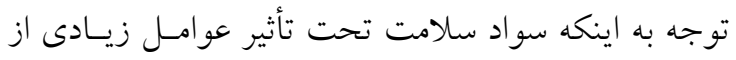
جمله عوامل فردى (مانند سن، جنسيت، نزاد، تحصسيلات

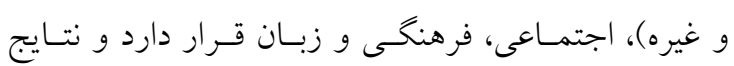

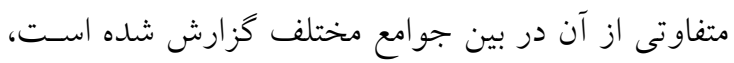

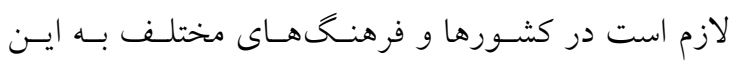
موضوع يرداخته شود (17). با توجه به اين كه بيمارى آسم تأثير عميقى بر روند برداند

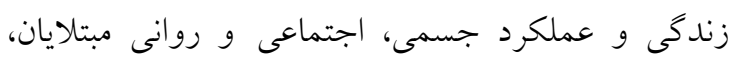

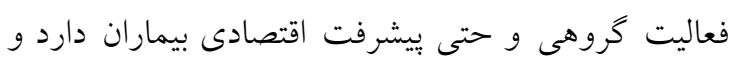

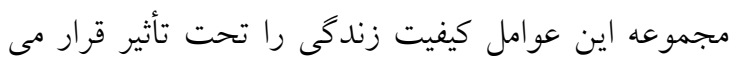

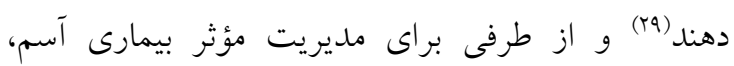

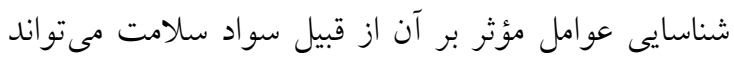

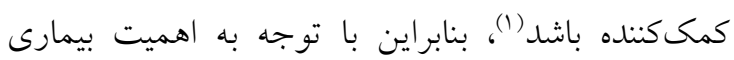

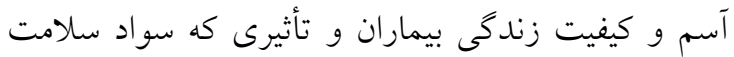
ممكن است بر آن بحذارد، اين مطالعه با هدف تيمارن تعيين

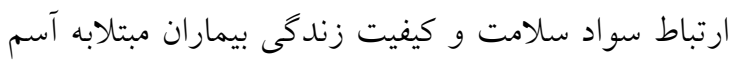

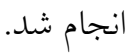

\section{مقدمه}

آسم بيمارى مزمن راه هوايى است براى تمام كـروههـاى

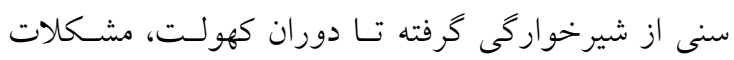

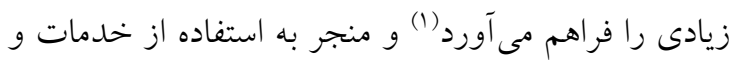

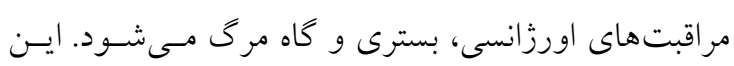

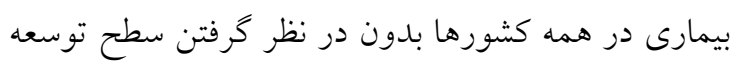

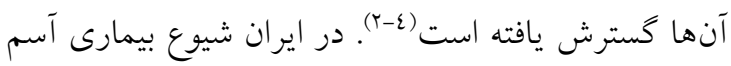

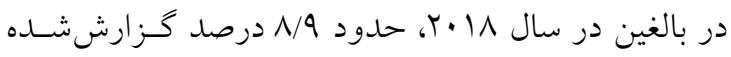
است (0). درمان و كتترل بيمارى آسم طولانى مدت بل بودهه و

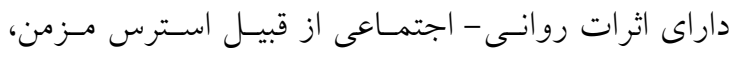
نغرانى نسبت به آينده، مشكلات خانو ادكى و شغلى و بار

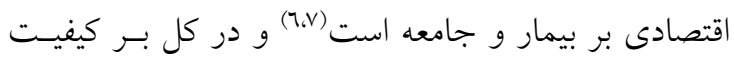

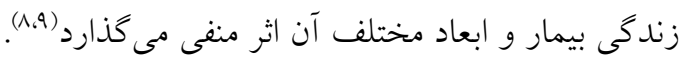

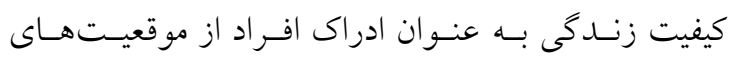
زندگى، ارزش هاى آنها، اهداف، انتظارات، استانداردها و

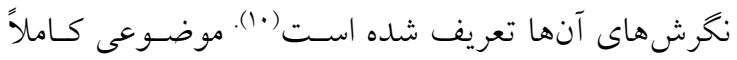

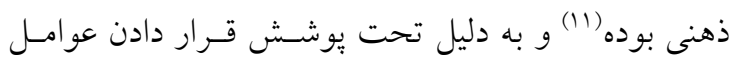

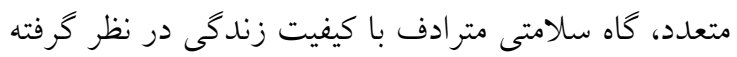

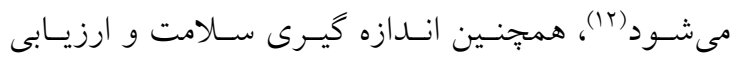

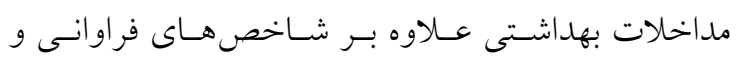

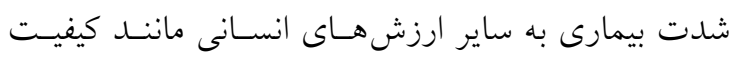

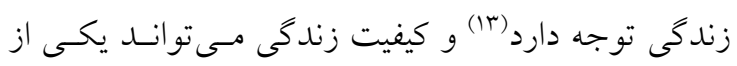

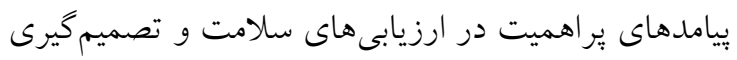

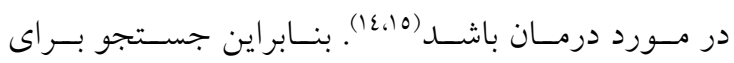

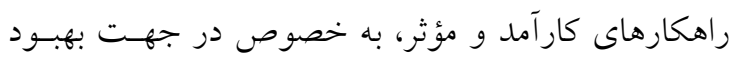

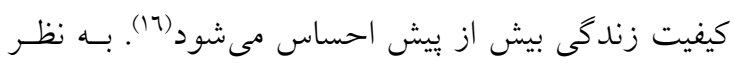

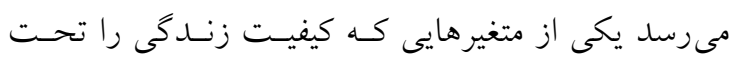

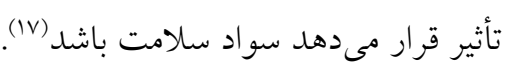
سواد سلامت شامل مجموعهاى از مهارتهـاى خوانـدن،

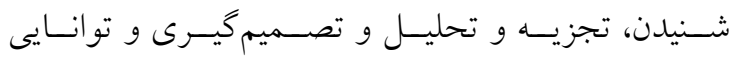

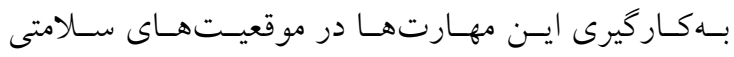

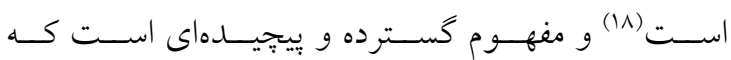
مهـارتهـاى شـناختى اجتمــاعى و توانـايى افــاد بــراى دستيابى بـه درى و اسـتفاده از اطلاعـات موجـود در راه 
بود. بر اين اساس نمــره كلـى بـهدسـتآمــده بــراى ايـن

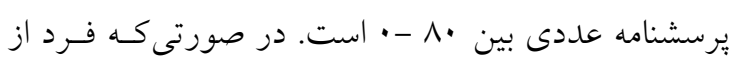

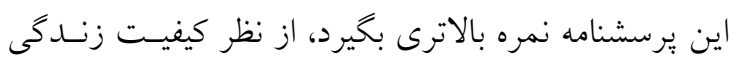

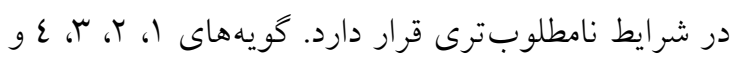

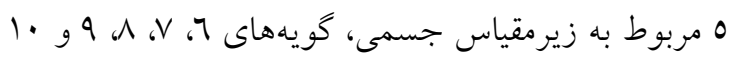

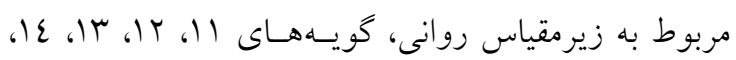

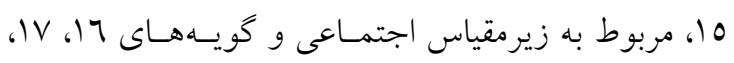

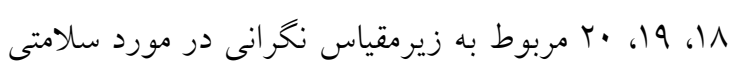

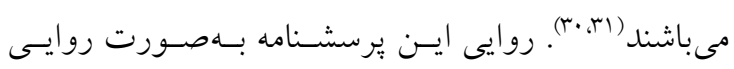

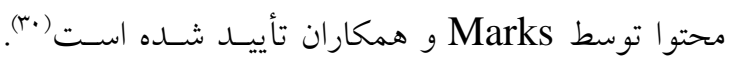
روايى نسخه فارسى با روش تعيين شاخص روايى محتـوا

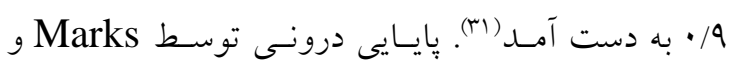

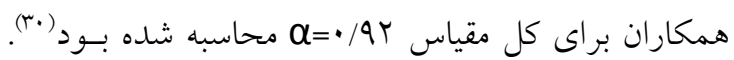

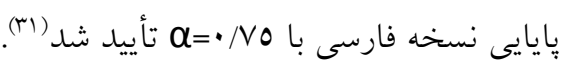

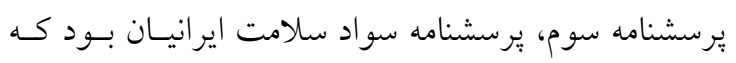

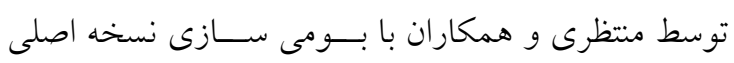

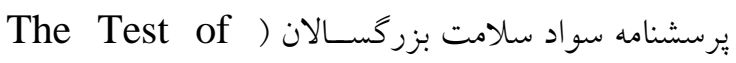
Functional Health Literacy in Adults: (TOFHLA ابتداى برسشنامه سؤالى در مورد نحــوه دريافـت مطالـبـ

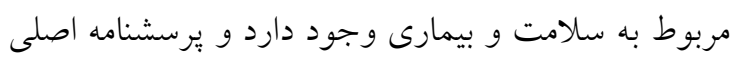

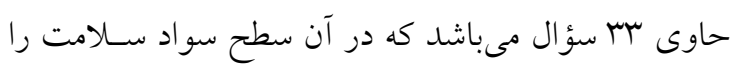

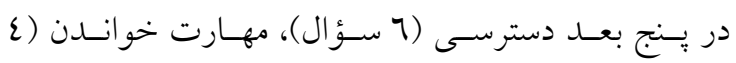

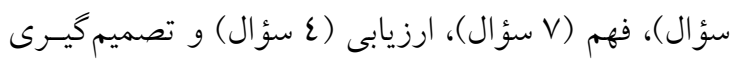

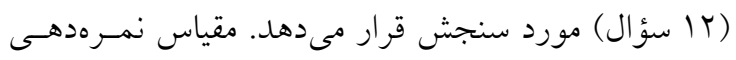
اين برسشنامه به صورت ليكرت بينج گزينهاى است. بدين صورت كه در سؤالات مربوط به مهارت خواندن؛ امتياز 0

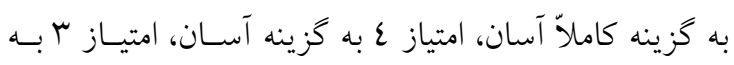

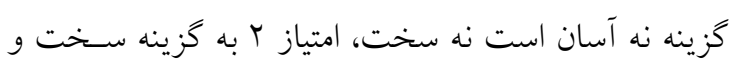

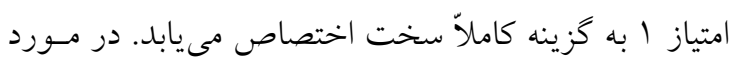

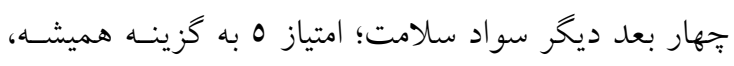

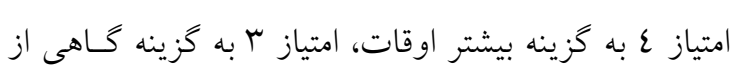

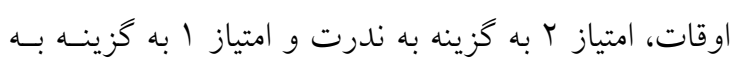

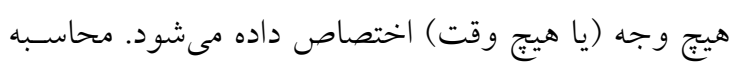

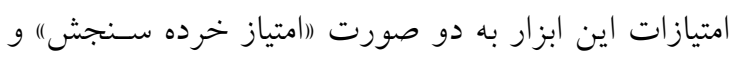

روش بروسى

اين يزووهش يك مطالعه تو صيفى از نوع همبستخى است.

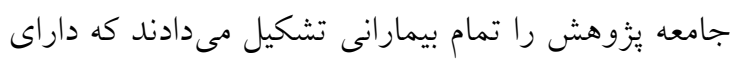

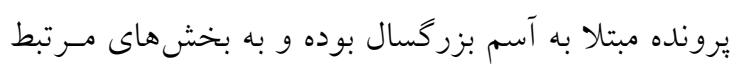

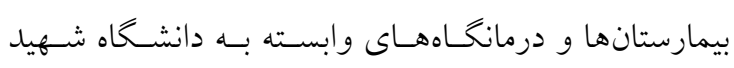
صدوقى يـزد در سـال لوها از آذر مـاه السى اسـفند مـاه

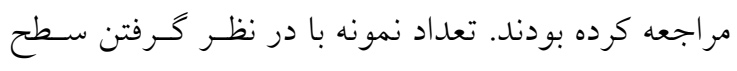

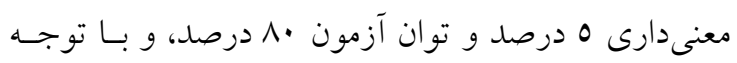

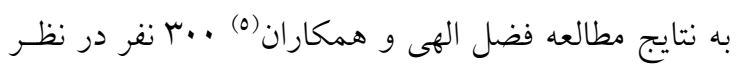

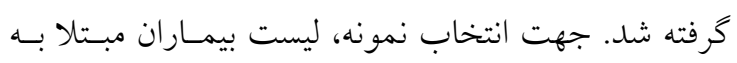

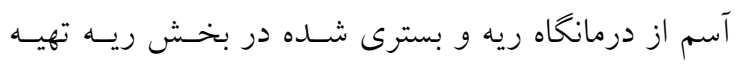

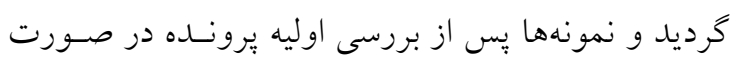
واجد شرايط بودن به روش نمونسه گيـرى تصـادفى سـاده

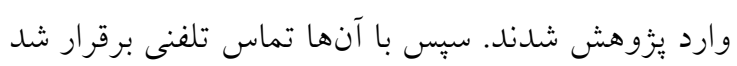

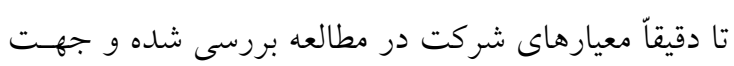

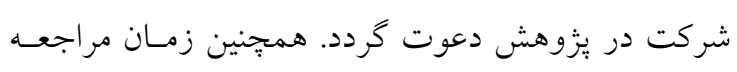

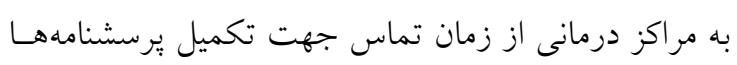

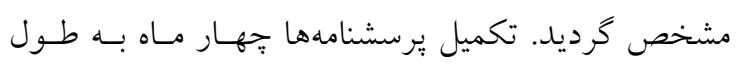

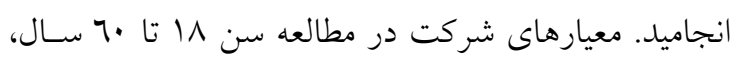

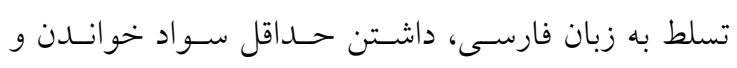

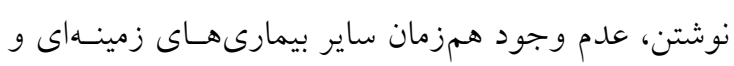

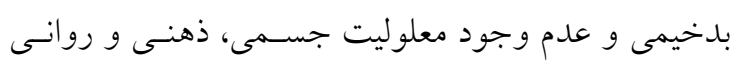

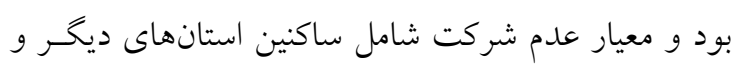
يا مراجعه نكردن به مر اكز درمانى در خند ماه آينده بود.

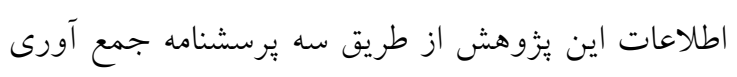

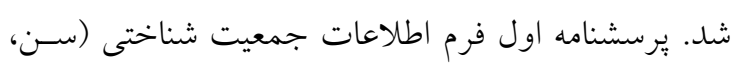

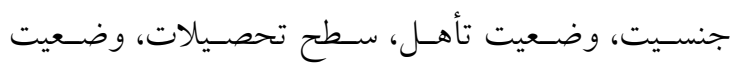

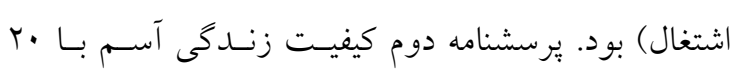

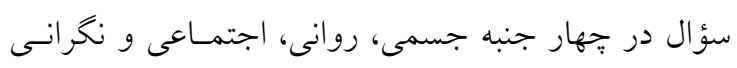

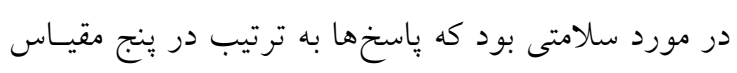

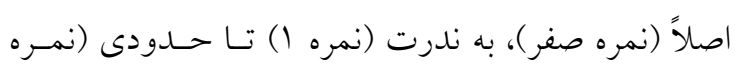

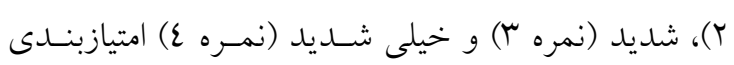
شدند. بدين ترتيب كه در همه حيطهها حسداكثر امتيازهـا،

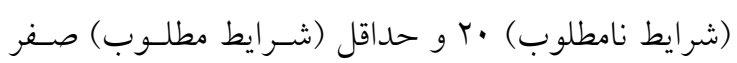




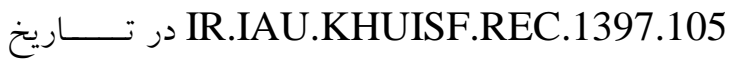

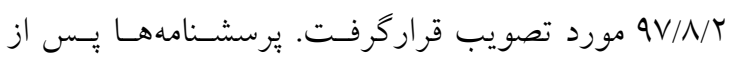

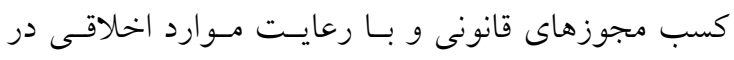

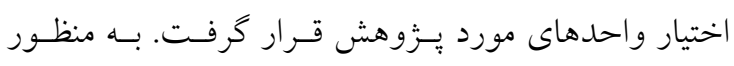

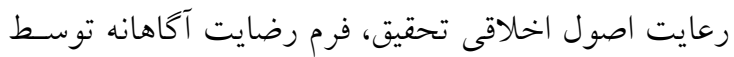

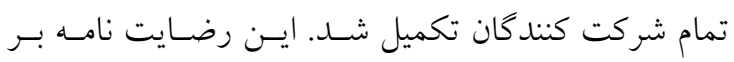

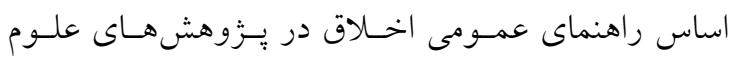
يزشكى تدوين شده بود. علاوه بـر ايسن، محرمانسه بـودن

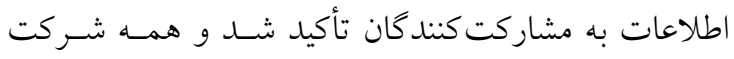
كنند كان رضـايت خــود را بــراى ورود بـهـ مطالعـه اعـلام كردند. تمام مشاركت كنندكان از اهداف تحقيق و ماهيـت داوطلبانه مشاركت آنان مطلع بودند. به آنها كفته شد كـهـ مى تو انند در هر مرحله از مطالعه شركت كنند.

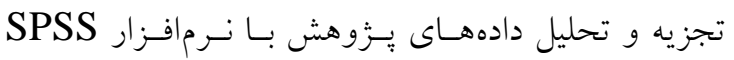

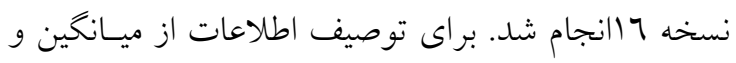
انحراف معيار، فراوانى مطلق و نسبى استفاده كرديد. قبـل

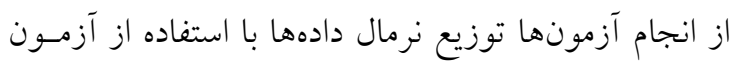

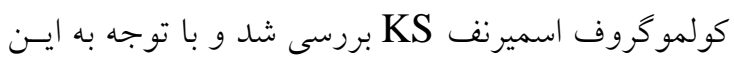

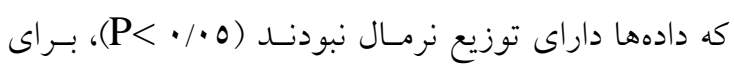
تعيين ارتباط بين متغيرها از آزمون آمارى اسبيرمن استفاده تراه

\section{يافتهها}

يافتهاى يُزوهش نشان داد بيشتر بيماران شركت كننده در

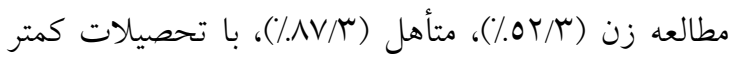

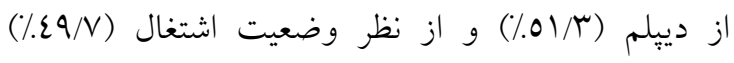

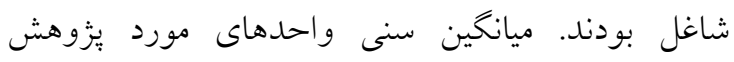

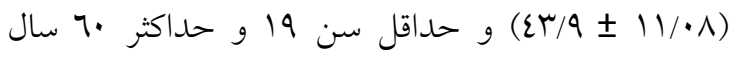

$$
\text { بود (جدول شماره ()). }
$$

(امتياز كل)" مى باشد، بدين صورت كـه نمــره ايسـن ابـزار از

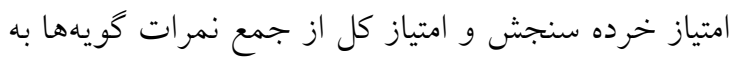

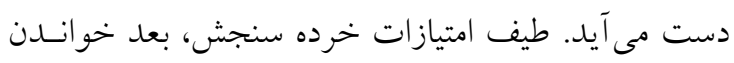

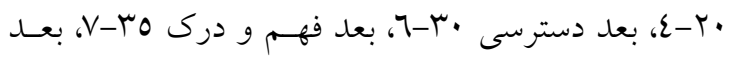

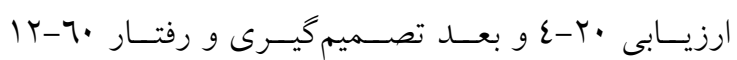

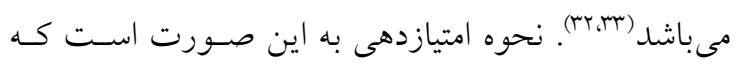

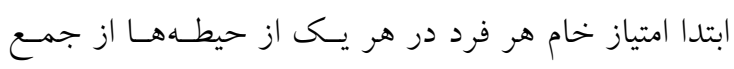

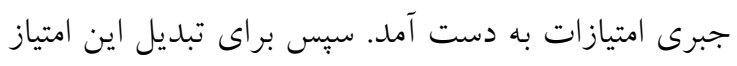

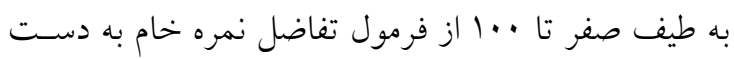

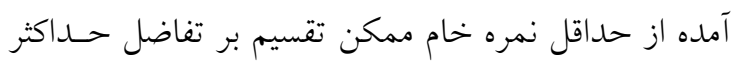

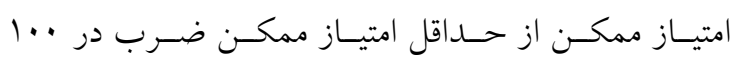

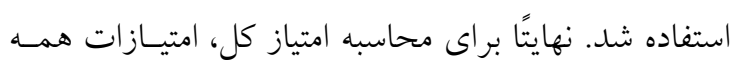
ابعاد (بر اساس طيف صفر تا ...1) جمع شده و بر تعـداد

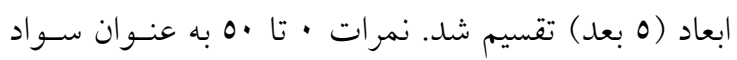

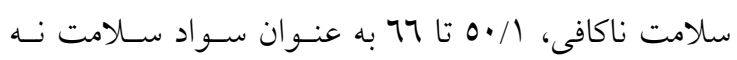

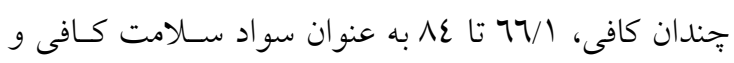

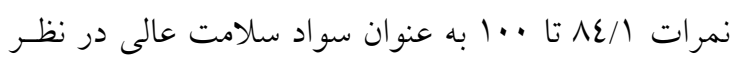

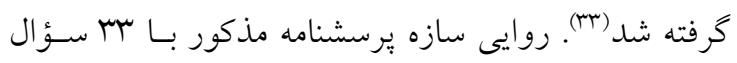

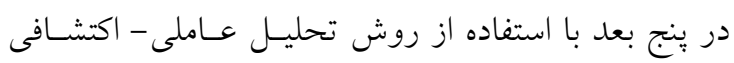

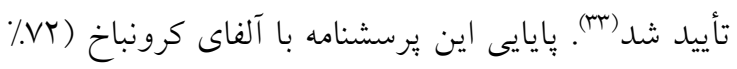

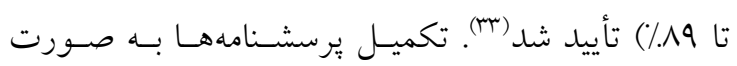

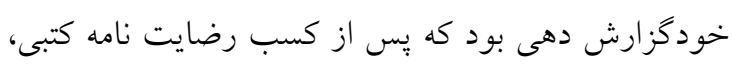

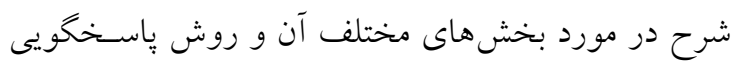

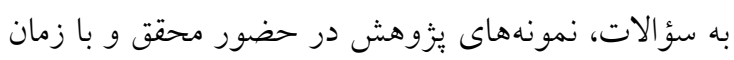

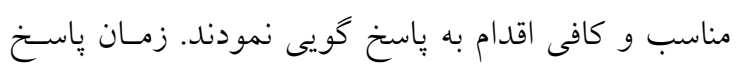

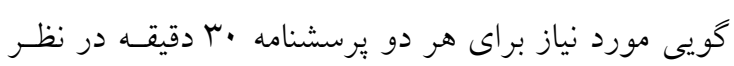
كرفته شد.

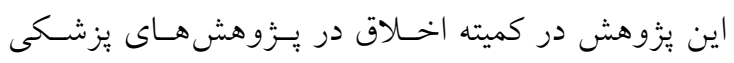

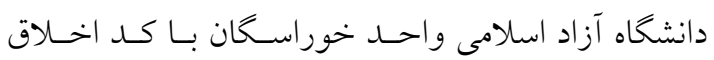


جدول شماره ا: توزيع فراوانى مطلق و نسبى واحدهاى مورد يزوهش بر حسب مشخصات جمعيت شناختى

\begin{tabular}{|c|c|c|c|c|c|c|}
\hline \multicolumn{2}{|c|}{ سواد سلامت } & \multicolumn{2}{|c|}{ كيفيت زندكى } & \multirow{2}{*}{ تعداد (درصد) } & \multirow{2}{*}{\multicolumn{2}{|c|}{ متغير }} \\
\hline نتيجه آزمون & انحراف معيار 土 ميانخين & نتيجه آزمون & انحراف معيار 土 ميانخين & & & \\
\hline $\mathrm{Z}=-\cdot / \varepsilon \mathrm{V}$ & $1 \cdot \cdot / \cdot 7 \pm r N / 7 r$ & $\mathrm{Z}=-\cdot / \wedge \Lambda \mid$ & $\varepsilon\rceil / N \Psi \pm \varepsilon / 9 \Lambda$ & $(0 \mathrm{~T} / \mathrm{T}) \backslash 0 \mathrm{~V}$ & زن ان & جنس \\
\hline $\mathrm{P}=\cdot / 7 \pi$ & $1 \cdot 1 / 01 \pm r / / 07$ & $\mathrm{P}=\cdot / r V$ & $\varepsilon V / 1 \cdot \pm 0 / 7\rceil$ & $(\varepsilon V / V) \backslash \varepsilon r$ & مرد & \\
\hline$Z=-7 / r$ & $q V / \backslash \varepsilon \pm Y N / \cdot q$ & $\mathrm{Z}=-\cdot / 0$ & $\varepsilon \bigvee / \Lambda \Lambda \pm 0 / \varepsilon \wedge$ & $(\Lambda V / r) r \neg r$ & متأهل & وضعيت \\
\hline $\mathrm{P}<\bullet / \cdot \cdot 1$ & $1 T O / 9 T \pm 17 / N$ & $\mathrm{P}=\cdot / 71$ & $\varepsilon V / \backslash \wedge \pm \varepsilon / \cdot \Gamma$ & $(I T / V) \mu_{\Lambda}$ & مجرد & ت تأهل \\
\hline$X^{2}=9 \Lambda / 9 \varepsilon$ & $10 / V 0 \pm$ Y0/07 & $\mathrm{X}^{2}=1 / \Lambda \mu$ & $\left.\sum V / \Gamma\right) \pm 0 / \Gamma q$ & $(01 / \pi) 10 \varepsilon$ & زير ديبلم & سطح \\
\hline$d f=r$ & $11 \cdot / 0 r \pm r r / 9 \varepsilon$ & $d f=r$ & \{\urcorner$/ V\urcorner \pm\{/ q 1$ & $(T \wedge / \Gamma) \wedge 0$ & دييلم & تحصيلات \\
\hline $\mathrm{P}<\bullet / \cdot \cdot 1$ & $|r O / \cdot \Lambda \pm| T / \Lambda r$ & $\mathrm{P}=\cdot / \mu q$ & $\varepsilon 7 / \mu\urcorner \pm 0 / 70$ & $(Y \cdot / \varepsilon) 71$ & كارشناسىو كارشناسىارشد & \\
\hline$X^{2}=v \varepsilon / \Gamma_{q}$ & $\| r / 09 \pm r \varepsilon / 1 V$ & $X^{2}=1 / N_{0}$ & $\varepsilon V / 19 \pm 0 / 0 \varepsilon$ & $(\varepsilon q / v) \mid \varepsilon q$ & شاغل & \\
\hline$d f=r$ & $q \cdot / r q \pm r r / 7 r$ & $d f=r$ & $\{\tau / 0 \cdot \pm 0 / T\}$ & $(\mathrm{rol} \cdot)) 1 \cdot 0$ & خانهدار & وضعيت \\
\hline \multirow[t]{2}{*}{$\mathrm{P}<\bullet / \cdot \cdot 1$} & $V \cdot / A V \pm 1 / N / 07$ & $\mathrm{P}=\cdot / \varepsilon)$ & $\varepsilon V / 0 . \pm 0 / .7$ & $(1 \cdot / V) r T$ & بازنشسته & اشتغال \\
\hline & $\mid r r / l \varepsilon \pm r \cdot / V A$ & & $\varepsilon 0 / \nearrow \varepsilon \pm \varepsilon / \cdot r$ & $(\varepsilon / V) \backslash \varepsilon$ & دانشجو & \\
\hline Rho $=-$ & N7 $\quad \mathrm{P}<\cdot / \cdot \cdot 1$ & Rho $=-$ & $\mathrm{P}=\cdot / \Lambda r$ & $\{r / q \mid \pm 11 / \cdot 1$ & انحراف معيار 土 ميانخين & 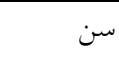 \\
\hline
\end{tabular}

مواردى از قبيل تلفن گويا و كتابجه،، جزوه، بروشورهاى

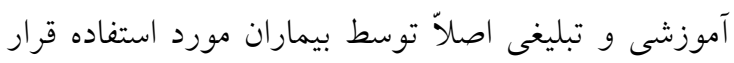

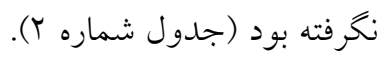

تحليل داده در مورد روشهاى دريافت مطالب مربوط به

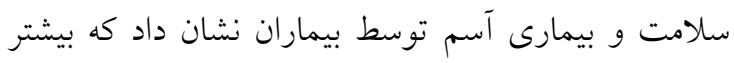

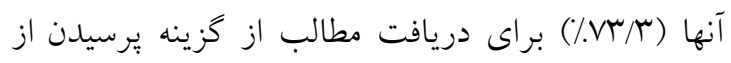

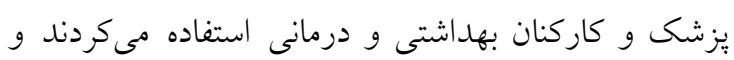

جدول شماره r: توزيع فراوانى مطلق و نسبى واحدهاى مورديزوهش بر حسب روش دريافت مطالب مربوط به سلامت و بيمارى

\begin{tabular}{|c|c|c|c|}
\hline تعداد (درصد) & تعداد (درصد) & عدم انتخاب كزينه & 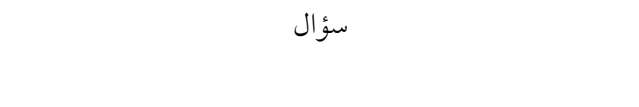 \\
\hline$(1 \cdots) \mu^{\prime} \cdot$ & $(V \pi / \mu) Y r$. & $(Y \backslash / V) \wedge \cdot$ & يرسيدن از يزشك و كاركنان بهداشتى و درمانى \\
\hline$(1 \cdots) \mu \cdots$ & $(11 / \Gamma) \mu \varepsilon$ & $(\Lambda \Lambda / V) Y 7 \neg$ & 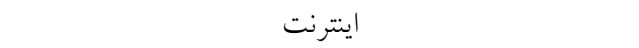 \\
\hline$(1 \cdot \cdot) \mu^{\prime} \cdot$ & $(\cdot / \cdot)$ & $(1 \cdots) \mu_{\cdots}$ & ت تلفن گويا \\
\hline$(1 \cdot \cdot) \mu \cdot$ & $(1 \varepsilon / \cdot) \varepsilon r$ & 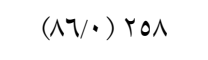 & راديو و تلويزيون \\
\hline$(1 \cdots) \mu \cdots$ & $(\cdot / \mu) 1$ & $(99 / V) r 99$ & روزنامه، نشريه و مجلات \\
\hline$(1 \cdots) \mu \cdots$ & $(9 / \cdot) r V$ & $(91 / \cdot) r V r$ & ير يرسيدن از دوستان و آشنايان \\
\hline$(1 \cdot \cdot) \mu_{*}$ & $(\cdot / \cdot) \cdot$ & $(1 \cdots) \mu_{\cdots}$ & كتابجه، جزوه، بروشورهاى آموزشى و تبليغى \\
\hline$(1 \cdots) \mu$ & $(\cdot / \mu) 1$ & $(99 / V) r 99$ & 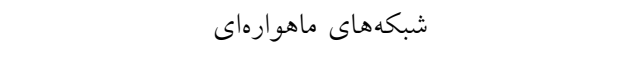 \\
\hline$(1 \cdot \cdot) \mu^{\prime} \cdot$ & $(Y I / V) 70$ & $(V \Lambda / r)$ rro & نمىدانم بايد از كجا اطلاعات مورد نيازم را به دست آورم \\
\hline
\end{tabular}

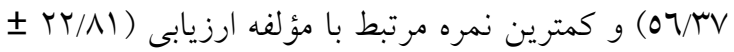
(ع/NV بالاترين رتبه عالى بودن (جr/) مربوط به مؤلفه خواندن و
يافتهها نشان داد كه ميانخين و انحر اف معيار سواد سلامت

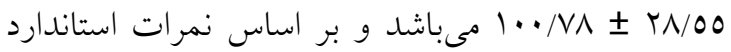
شده بيشترين نمره مربوط به مؤلفه دسترسى (•r/T I • 
داراى سطح ناكافى از سواد سلامت مىباشند (جدول كمترين رتبه يا ناكافى بودن سواد سلامت مربوط به مؤلفه شماره (r) (1) (n)

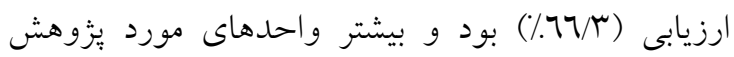

جدول شماره "ّ: ميانكَين، انحراف معيار و رتبدبندى سطح سواد سلامت و مؤلفههاى آن در واحدهاى مورد بزووهش

\begin{tabular}{|c|c|c|c|c|c|c|c|}
\hline \multirow{2}{*}{ نمره بر مبناى · تا +.1 } & انحراف معيار \ميانكين & عالى & كافى & نه جندان كافى & ناكافى & \multicolumn{2}{|c|}{ سواد سلامت } \\
\hline & & & & & & & \\
\hline \multirow[t]{2}{*}{$20 / 07 \pm \Upsilon N / \varepsilon\rceil$} & $11 / r 9 \pm \varepsilon / 00$ & $1 \cdot 1$ & 01 & 0 & $1 \% 7$ & تعداد & خواندن \\
\hline & $\varepsilon-r$. & $\mathrm{rq} /$ & $\mathrm{IV} / \cdot$ & $1 / V$ & $\varepsilon 0 / \pi$ & درصد & \\
\hline \multirow[t]{2}{*}{$\Delta T / r V \pm r T / 7}$. & $19 / 0 r \pm V / A r$ & $r r$ & $v r$ & rv & $17 V$ & تعداد & دسترسى \\
\hline & ૧-r. & $\mathrm{V} / \mathrm{V}$ & $r \varepsilon / \Gamma$ & $1 \pi \mu$ & $00 / \mathrm{V}$ & درصد & \\
\hline \multirow[t]{2}{*}{$00 / 1 V \pm r T / N V$} & $r Y / \Sigma 0 \pm V / \varepsilon q$ & $\varepsilon v$ & $v q$ & $\mu$ & $|\varepsilon|$ & تعداد & فهم و درك \\
\hline & v-ro & $10 / \mathrm{V}$ & $r \eta / r$ & $11 \%$ & $\varepsilon \mathrm{V} / \cdot$ & درصد & \\
\hline \multirow[t]{2}{*}{$\varepsilon \cdot / V V \pm r Y / \Lambda 1$} & $1 \cdot / 0 r \pm r / \neg \varepsilon$ & v & $\sum 7$ & $\sum \wedge$ & 199 & تعداد & ارزيابى \\
\hline & $\varepsilon-19$ & $r / \mu$ & $10 / r$ & $17 /$ & $77 / r$ & درصد & \\
\hline \multirow[t]{2}{*}{$0 Y / \cdot 7 \pm 10 / 1 \mathrm{~V}$} & $r \checkmark / 99 \pm V / r \Lambda$ & $r$ & $0 \wedge$ & 91 & IET & تعداد & تصميم كيرى \\
\hline & YI-or & $\cdot / v$ & $19 / \pi$ & rTN & $\varepsilon V / r$ & درصد & \\
\hline \multirow[t]{2}{*}{$01 / r 0 \pm r / / \pi$} & $\ldots / V \Lambda \pm r N / 00$ & 11 & $\wedge \varepsilon$ & $\pi$ & 1ro & تعداد & كل \\
\hline & $\varepsilon \varepsilon-10 r$ & $\mathrm{~T} /$ & r & rr & so & درصد & \\
\hline
\end{tabular}

بعد نخرانى در مورد سلامتى (IV/Z) I Y Y بود (جدول شماره ع)
نتايج نشان مىدهد كه ميانگين كيفيت زندكى بيماران

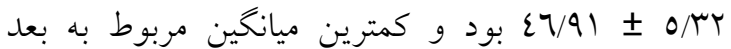

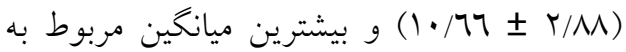

جدول شماره ع: ميانكين و انحراف معيار كيفيت زندگى و مؤلفههاى آن در واحدهاى يزوهش

\begin{tabular}{|c|c|c|c|c|c|}
\hline حداكثر نمره يرسشنامه & حداقل نمره برسشنامه & انحر اف معيار & ميانكين & تعداد كويه & كيفيت زندگى \\
\hline$r$. & . & $r / M$ & $1 . / 77$ & 0 & جسمى \\
\hline r. & . & $r / 79$ & $1 . / \mathrm{VV}$ & 0 & 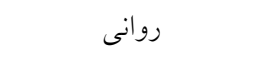 \\
\hline r. & . & r/ro & $17 / 11$ & 0 & اجتماعى \\
\hline$r \cdot$ & . & $r / M$ & $|V / \varepsilon|$ & 0 & نخر انى در مورد سلامتى \\
\hline ᄉ. & . & ORT & $\{7 / 9\}$ & r. & كيفيت زندگى كل \\
\hline
\end{tabular}

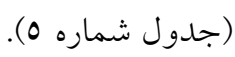

نتايج آزمون اسـبيرمن حساكى از آن بـود كـهـ بـين سـواد

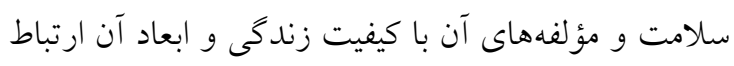
آمارى معنى دارى وجود نداثت. 
جدول شماره 0: همبستَى سواد سلامت و مؤلفههاى آن با كيفيت زندتى و مؤلفههاى آن

\begin{tabular}{|c|c|c|c|c|c|c|c|c|c|c|}
\hline \multicolumn{2}{|c|}{ كيفيت زندكى } & \multicolumn{2}{|c|}{ نخرانى در مورد سلامتى } & \multicolumn{2}{|c|}{ اجتماعى } & \multicolumn{2}{|c|}{ 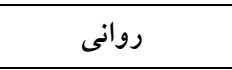 } & \multicolumn{2}{|c|}{ جسمانى } & \multirow{2}{*}{ ختغواندن } \\
\hline $.107 \varepsilon$ & . & $\cdot N\urcorner \wedge$ &.$/ \cdot 1 V$ &.$/ 7 V V$ & $\% \cdot r \varepsilon$ & $\cdot / 7 V$ &.$/$ YO & $\cdot / \wedge r$ &.$/ .14$ & \\
\hline$\cdot / 99 \mathrm{~V}$ & $\cdot \cdots 1$ & $\cdot / N\urcorner \varepsilon$ &.$/ \cdot 1 V$ & $\cdot / 7 \varepsilon 1$ & $\cdot / r V$ & $\cdot / v \cdot V$ &.$/ r r$ &.$/ 11$ &.$/ .94$ & دستر د د \\
\hline$\cdot / \mathrm{Va}$ & $\cdots \cdots \wedge$ &.$/ 79$ & 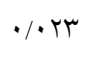 &.$/ 91 \varepsilon$ & 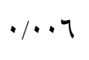 &.$/ 909$ & $\cdot / \cdot r$ & - KN & $.1 .7 r$ & فهم و درى \\
\hline.$/ 107$ & $\cdot / \cdot \Lambda r$ & $\cdot / 297$ & $\cdot / \cdot r q$ & . /9ro & $\%$ & $\cdot / 9 \varepsilon 7$ & $\cdot / \cdot \varepsilon$ & $\cdot / T M$ & $.1 .7 r$ & ارزيابى \\
\hline$\cdot / \cdot r$ &.$/ r r$ &.$/ 91 \mathrm{~V}$ & 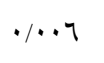 &.$/ 9 \vee 9$ & $\% r$ & $\cdot / A \cdot \varepsilon$ &.$/ \cdot 1 \varepsilon$ & $\cdot / 2 r q$ & $\%$ \% & تصميم كيرى \\
\hline$\cdot / 7 \mathrm{VV}$ & $\%$ & $\cdot / 117$ &.$/ .14$ &.$/ 9 V 1$ & $\% r$ &.$/ 910$ &.$/ .7$ & $\cdot / \varepsilon 7 \varepsilon$ & $\% \varepsilon r$ & سواد سلامت \\
\hline
\end{tabular}

رتبهبندى، سواد سلامت بيشتر واحدهاى مورد يزوهش در

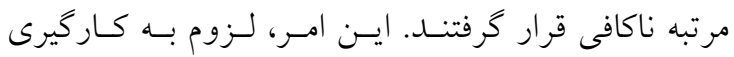

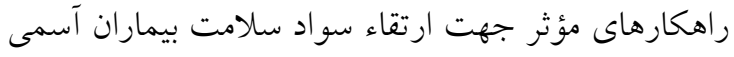
را نشان مى دهد جرا كه سواد سلامت كافى براى مشاركت بيمار در نظامهـاى مراقبـت بهداشـتى و اخـــ تصـميمات

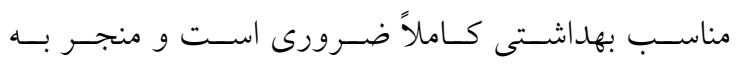
توانمندسازى افراد در به كار بستن اطلاعات و دستورهاى مربوط به سلامت مى شود كه با توجه به ناكافى بودن سواد

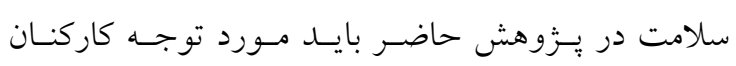

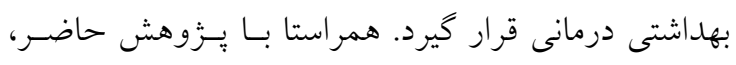

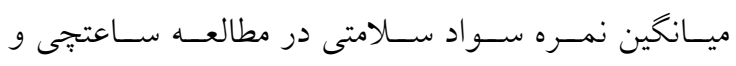

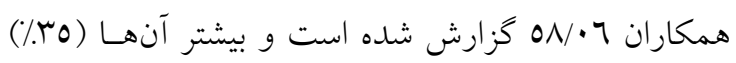

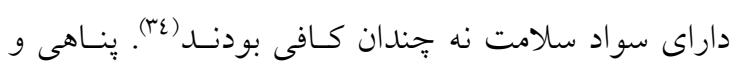

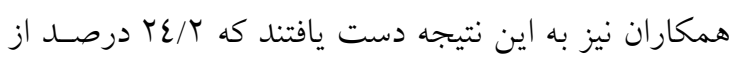

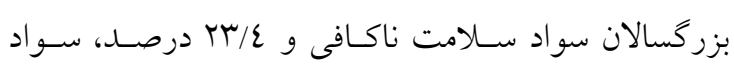

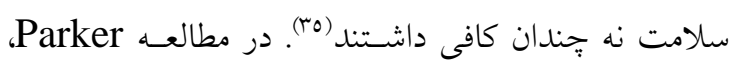

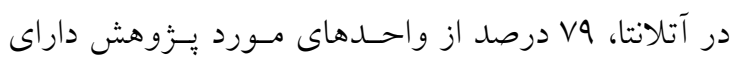

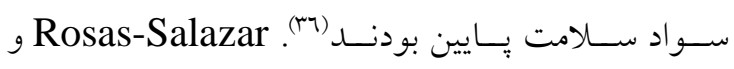

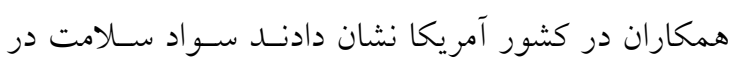

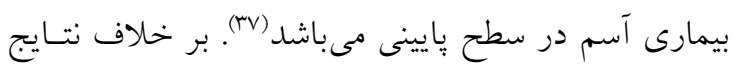

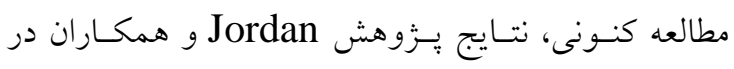

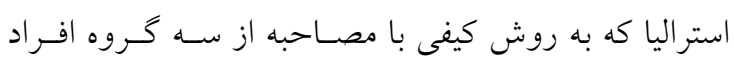
داراى بيمارى مزمن، جمعيت عادى و افراد مراجعه كننــهـ

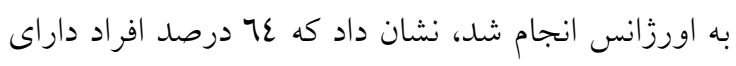

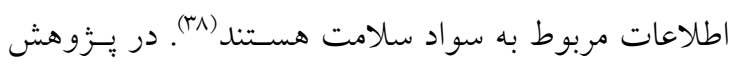

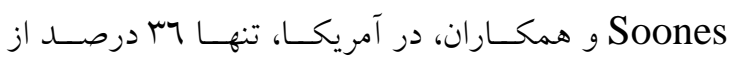
بيماران سواد سلامت ناكافى داشـتند و در آنهـا احتمـال
همان طور كه در جدول شماره ا مشخص شده است بين

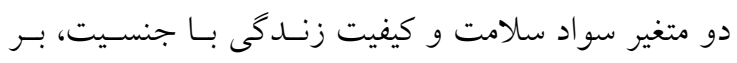

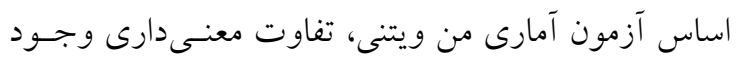

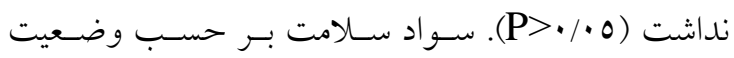

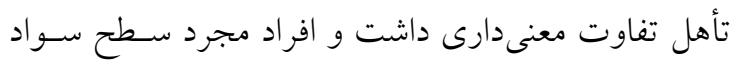

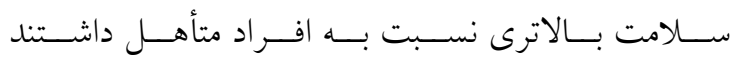

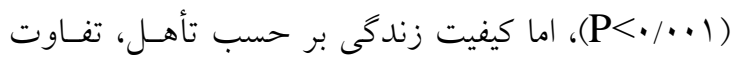

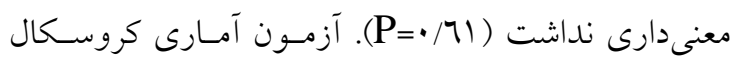
واليس بين سواد سلامت با سطح تحصسيلات و وضعيت

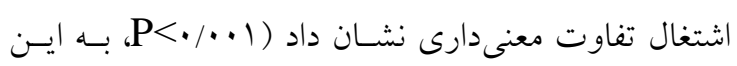
ترتيب كه سطح تحصيلات كارشناسى ارشد و كارشناسى

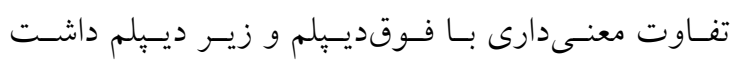
(P<•/・l)

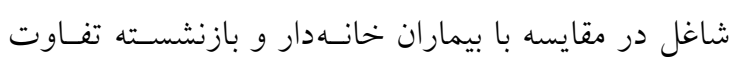

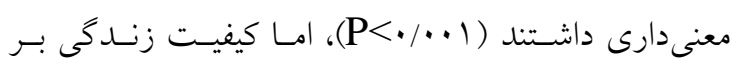

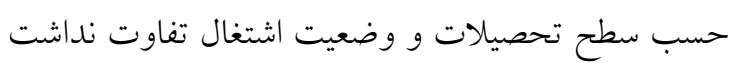

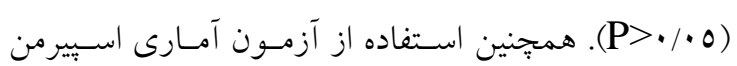

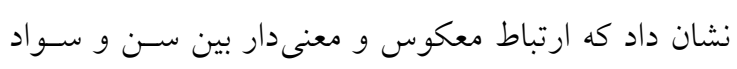

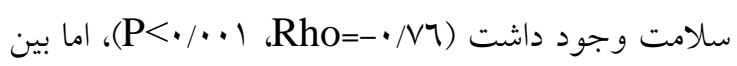
سن و كيفيت زندكى ارتبـاط معنسى دارى وجــود نداشـت . $(\mathrm{P}=\cdot$ /Nr Rho=-・/・1)

\section{بحث و نتيجه تيرى}

نتايج اين مطالعه نشان داد ميـانخين نمـره سـو اد سـلامت

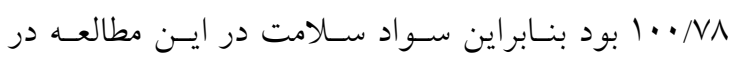

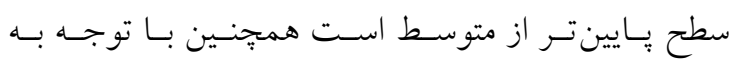


آسم كزارش كردند كه داشتن آسم به طسور قابـلتـو جهمى

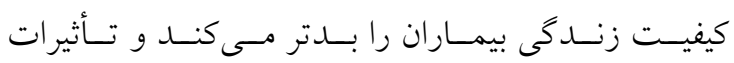

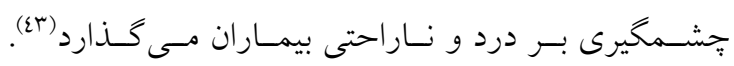

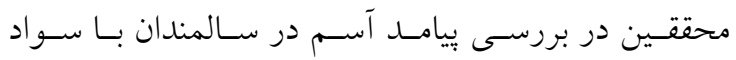

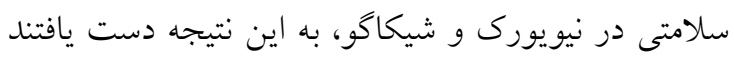

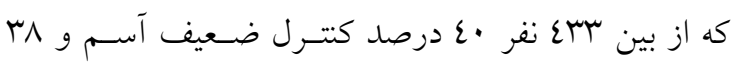

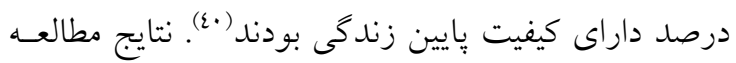

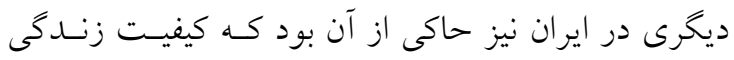

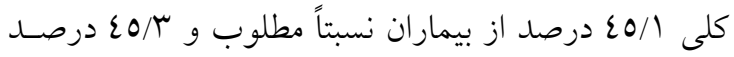

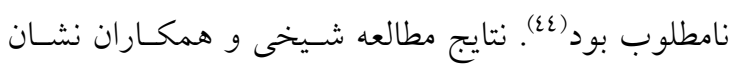
داد كه كيفيت زندكى كلى در بين افراد مبتلابه آسم بـايين

همجنِين يافتهها حاكى از اين بود كه بين سواد سـلامت و

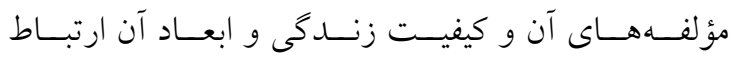

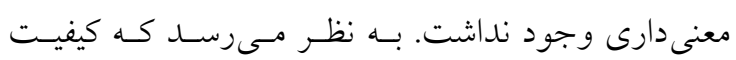

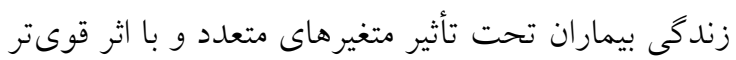

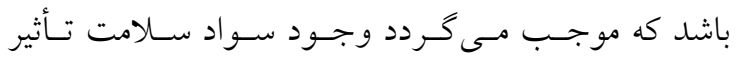

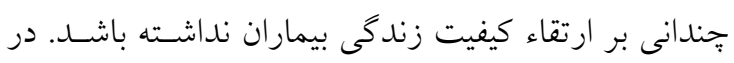

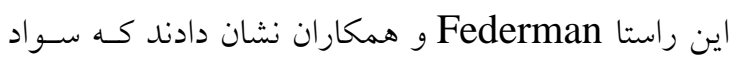

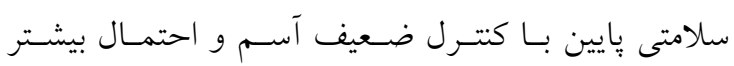

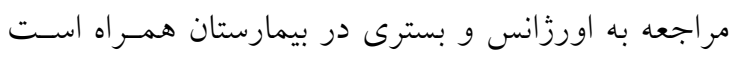

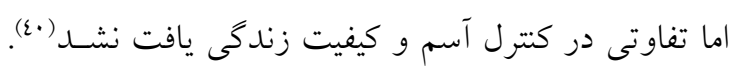

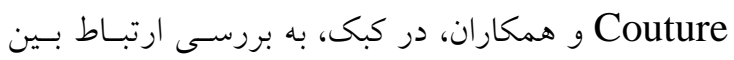

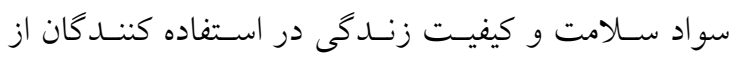
خدمات مراقبت بهداشتى اوليه كـه حسداقل يـك بيمـارى

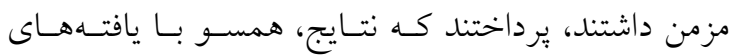
مطالعه حاضر بود و ارتباطى بـين سـواد سـلامت و ابعـاد

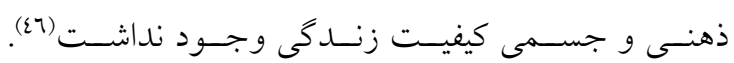

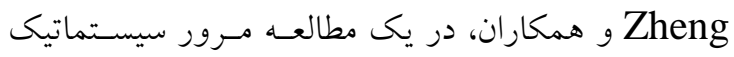
نشان دادند كه بين سو اد سلامتى با كيفيت زندكى به طـور متوسط همبستكى وجود دارد، ولسى نويسـندكان بيشــنهاد دادند كه كه اين يافته بايد توسط شواهد بيشترى يشتيبانى

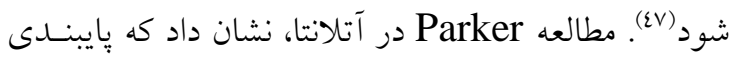

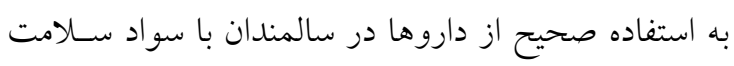

مصرف داروى آسم اشتباه و دريافـت بيشـتر دارو وجـود

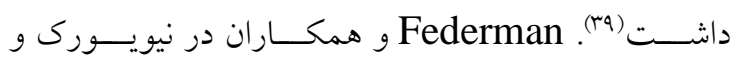

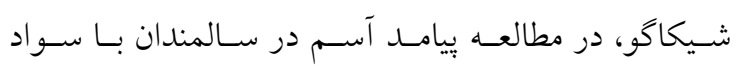

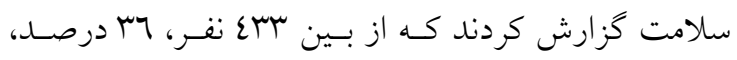

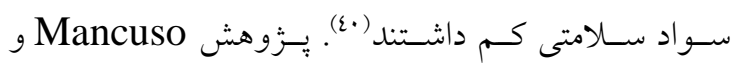
Rincon سـلامت مناسـب، 1 درصــ سـو اد سـلامت مـرزى و .

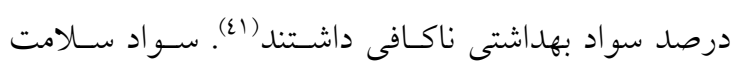

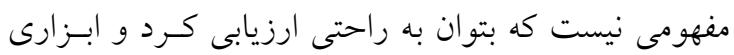
كه توافق جهانى داشته باشد معرفى نشده اسـت. بنـابراين بعيد نيست كه ابزارهاى مختلفى كه بـراى سـنجش سـواد سـلامت در يـزؤهش هـاى مختلـف مـورد اسـتفاده قـرار

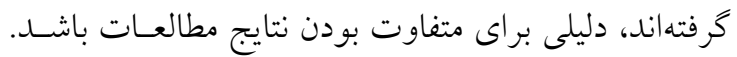

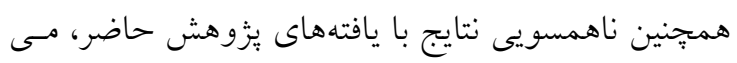

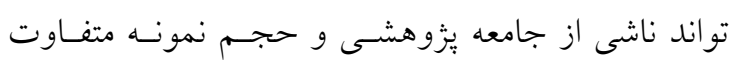

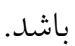

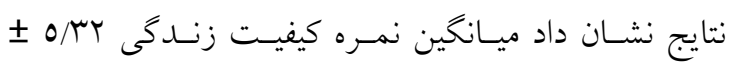

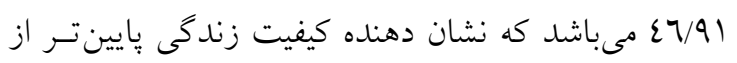

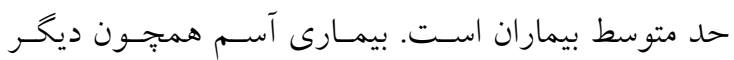

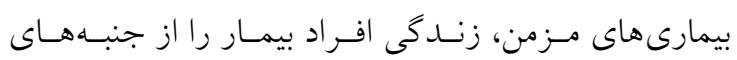

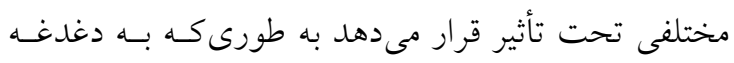

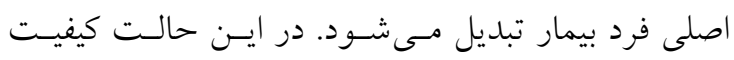

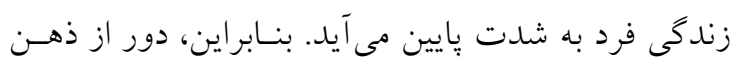

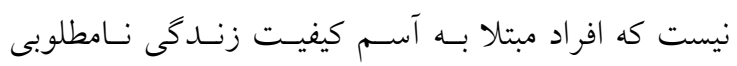

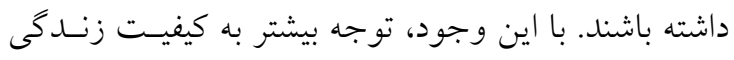

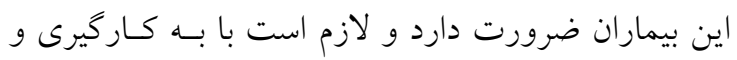

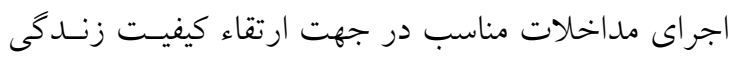

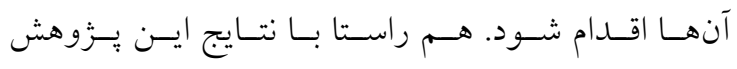
Urbstonaitis

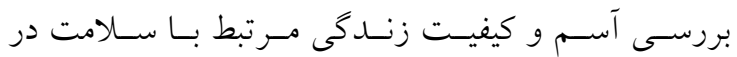
بزرگسالان در اواخر دوره ميانسالى برداختند، نشان دادند

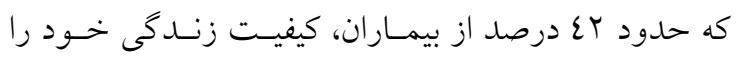

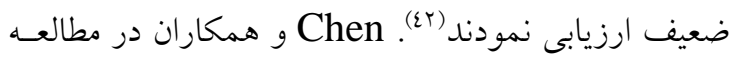
كيفيت زندكى مرتبط با سلامتى در كانادايىهاى مبـتلا بـهـ 
نيست كه اين متغيرها، تأثيرى بر هم ندارند. ممكن اسـت

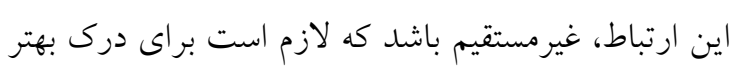

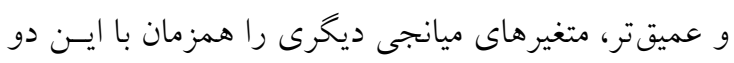
متغير ارزيابى نمود. بر اساس يافته هاى يززوهش سواد سلامت و مؤلفههاى آن (خواندن، فهم و درى، تصميم گيرى، دسترسى و ارزيابى)

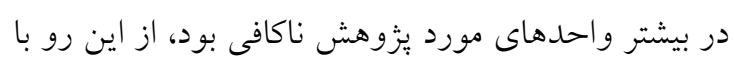

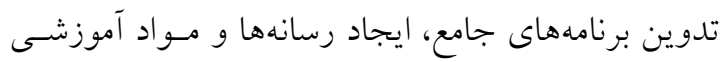

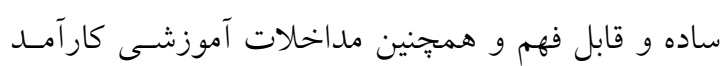
مىتوان كامى مؤثر جهت ارتقاى سلامت بيماران مبتلا بــهـ

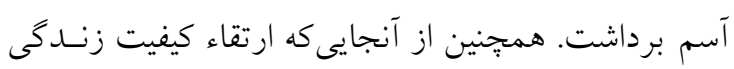

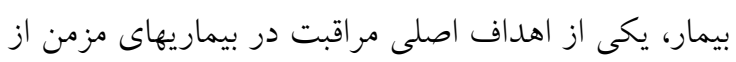

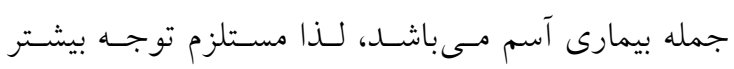

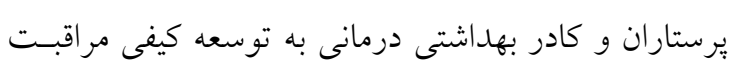

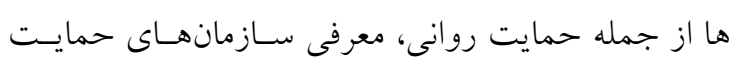

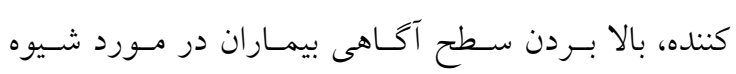
زندى و خودمر اقبتى مىباشد.

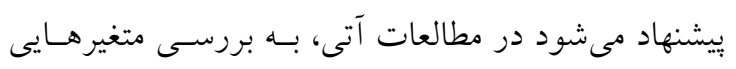

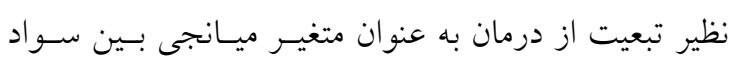

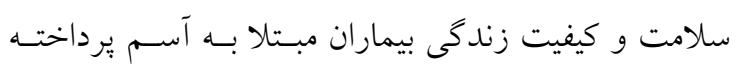

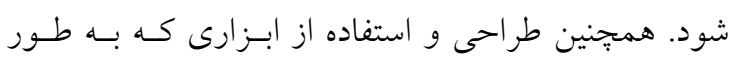

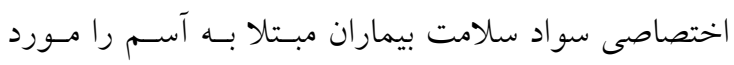

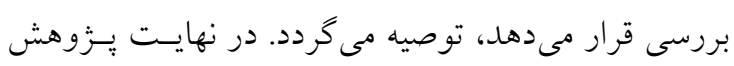

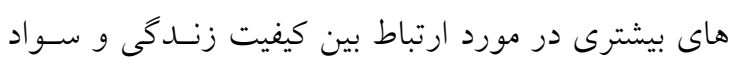
سلامت براى ساير كروههاى بيماران بيشنهاد مى دئرد. تعارض منافع:هيج گونه تعارض منافع توسط نويسندگان بيان نشده است.

\section{تقدير وتشكر}

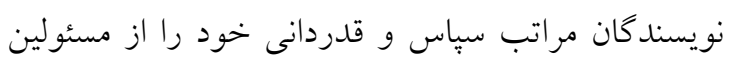

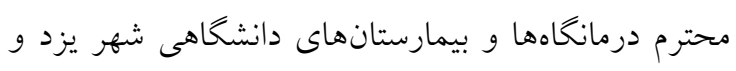
بيماران شركت كننده در مطالعه اعلام مىنمايند.

\section{References}

ڤايين بسيار كمتر از ديخر سالمندان است ("r7). Londoño در كشور سوئيس نشان داد سواد سـلامت بـالاتر بيمـاران

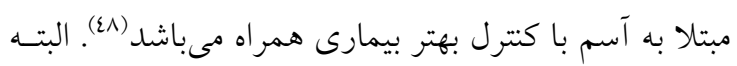
در مطالعه Londoño كيفيت زنـدكى بـهـ طـور مستقيم

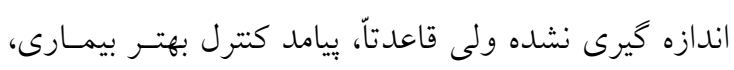

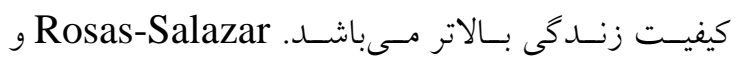

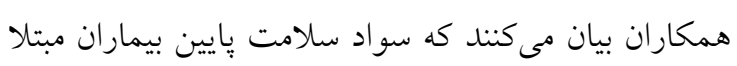

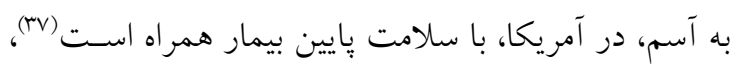
كه در اين مطالعه، سلامت به طور كلى مورد بررسى قـرار

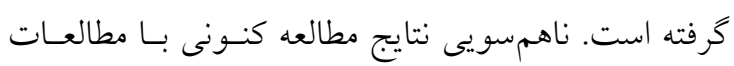

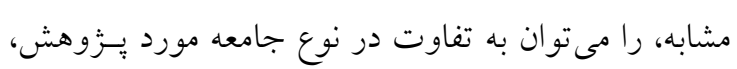

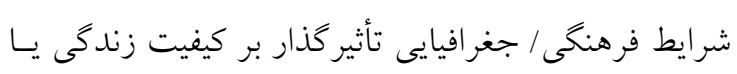

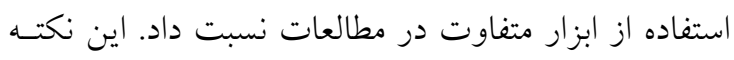

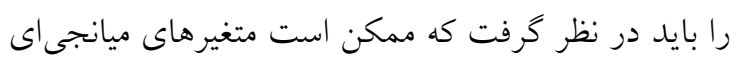

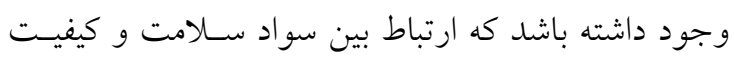

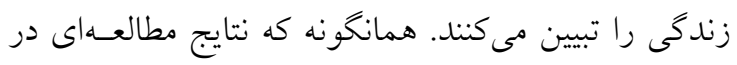

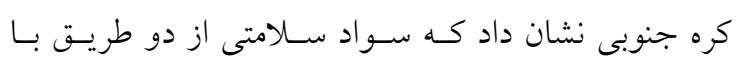
كيفيت زندكى مرتبط مىباشد اولاً سواد سلامتى در ارتباط مثبت با خواندن بر جسببهاى دارويى است، كه متعاقباً بـا تبعيت از درمان و كيفيت زندگى مرتبط است. ثانياً، سـواد

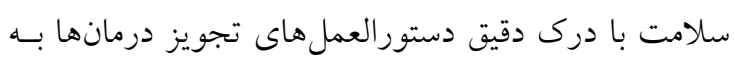

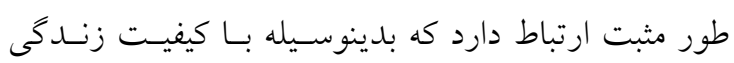
مرتبط مىباشد (29).

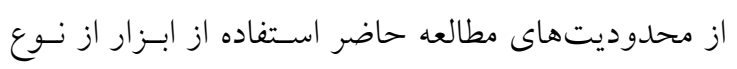

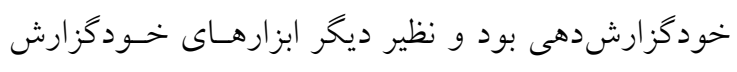
دهى، احتمال خستخى حسين تكميـل آن وجـود دارد كـهـ

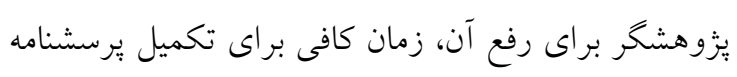

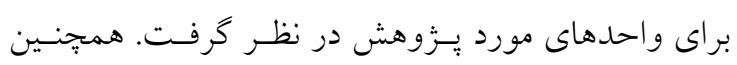
جهت امكان اجراى نمونه كيـرى تصـادفى فقـط بيمـاران

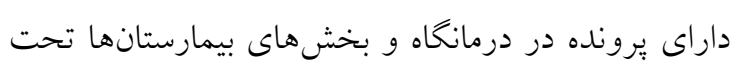
مطالعه قرار خرفتند.

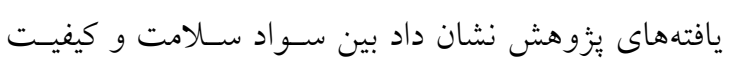

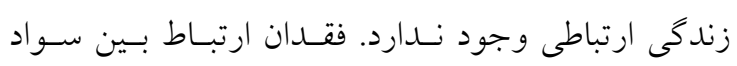

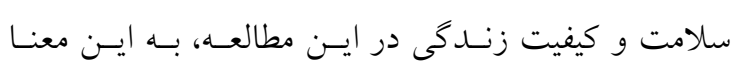


1. Lommatzsch M, Virchow CJ. Severe asthma: definition, diagnosis and treatment. Deuts Ärztebl Int. 2014;111(50):847-55.

2. Yektatalab SH, Ansarfard F. The relationship between mental health and quality of life in patients with Asthma. Psychiatric Nursing. 2014;2(1):64-72. [Persian]

3. O'Conor R, Wolf MS, Smith SG, Martynenko M, Vicencio DP, Sano M, Wisnivesky JP, Federman AD. Health literacy, cognitive function, proper use, and adherence to inhaled asthma controller medications among older adults with asthma. Chest. 2015;147(5):1307-15.

4. Solé D, Aranda CS, Wandalsen GF. Asthma: epidemiology of disease control in Latin Americashort review. Asthma Res Pract. 2017;3(1):4.

5. Fazlollahi MR, Najmi M, Fallahnezhad M, Sabetkish N, Kazemnejad A, Bidad K, Shokouhi Shoormasti R, Mahloujirad M, Pourpak Z, Moin M. The prevalence of asthma in Iranian adults: the first national survey and the most recent updates. Clin Respir J. 2018;12(5):1872-81.

6. Stanescu S, Kirby SE, Thomas M, Yardley L, Ainsworth B. A systematic review of psychological, physical health factors, and quality of life in adult asthma. NPJ Prim Care Respirat Med. 2019;29(1):1-11.

7. Baiardini I, Sicuro F, Balbi F, Canonica GW, Braido F. Psychological aspects in asthma: do psychological factors affect asthma management?. Asthma Res Pract. 2015;1(1):7.

8. Matsunaga NY, Ribeiro MA, Saad IA, Morcillo AM, Ribeiro JD, Toro AA. Evaluation of quality of life according to asthma control and asthma severity in children and adolescents. J Bras Pneumol. 2015;41(6):502-8.

9. Khassawneh B, Behbehani NH, Al-Jahdali HH, Al Qaseer AH, Gjurovic A, Haouichat H, Mahboub BH, Malvoti E, Naghshin R, Mackenzie S, Tabbal M. Quality of life in asthma patients from the Middle East and North Africa. InA53. ASTHMA EPIDEMIOLOGY 2018 May (pp. A1898-A1898). American Thoracic Society.

10. Pinto S, Fumincelli L, Mazzo A, Caldeira S, Martins JC. Comfort, well-being and quality of life: Discussion of the differences and similarities among the concepts. Porto Biomed J. 2017;2(1):612.

11. Bowling A. Measuring health: a review of subjective health, well-being and quality of life measurement scales. $4^{\text {nd }}$ ed. London: Open University Press; 2017.

12. Hosieni F, Mirzaei T, Ravari A, Akbary A. The relationship between health literacy and quality of life in retirement of Rafsanjan University of Medical Sciences. Journal of Health Literacy. 2016;1(2):92-9. [Persian]

13. Tay TR, Lee JW, Hew M. Diagnosis of severe asthma. Med J Aust. 2018;209(2 Suppl):S3-10.

14. Felce D, Perry J. Quality of life: Its definition and measurement. Res Dev Disabil. 1995;16(1):5174.

15. Salman-Yazdi N, Ghasemi I, Salahshourian A, Haghani H. Comparative study on the quality of life amongst asthmatic boys and girls aged between 7-17 in Zanjan. Iranian Journal of Cardiovascular Nursing. 2012;1(1):24-9. [Persian]

16. Skevington SM, Lotfy M, O'Connell K2. The World Health Organization's WHOQOL-BREF quality of life assessment: psychometric properties and results of the international field trial. A report from the WHOQOL group. Quality of life Research. 2004;13(2):299-310.

17. Wang C, Kane RL, Xu D, Meng Q. Health literacy as a moderator of health-related quality of life responses to chronic disease among Chinese rural women. BMC women's health. 2015;15(1):34.

18. Kasemsap K. Challenges and implications of health literacy in global health care. InAdvanced Methodologies and Technologies in Medicine and Healthcare 2019;p:185-97. IGI Global.

19. Valerio MA, Peterson EL, Wittich AR, Joseph CL. Examining health literacy among urban African-American adolescents with asthma. Journal of Asthma. 2016;53(10):1041-7.

20. Sørensen K, Van den Broucke S, Fullam J, Doyle G, Pelikan J, Slonska Z, Brand H. Health literacy and public health: a systematic review and integration of definitions and models. BMC public health. 2012;12(1):80.: 1-13.

21. Mohseni M, Khanjani N, Iranpour A, Tabe R, Borhaninejad VR. The relationship between health literacy and health status among elderly people in Kerman. Iranian Journal of Ageing. 2015;10(2):146-55. [Persian]

22. Brownson RC, Baker EA, Deshpande AD, Gillespie KN. Evidence-based public health. Oxford university press; 2017 Jun 15. 
23. Lynch MA, Franklin GV. Health Literacy: An Intervention to Improve Health Outcomes. InStrategies to Reduce Hospital Mortality in Lower and Middle Income Countries (LMICs) and Resource-Limited Settings 2019 Sep 10. IntechOpen. Available from: https://www.intechopen.com/books/strategies-to-reduce-hospital-mortality-in-lower-and-middleincome-countries-lmics-and-resource-limited-settings/health-literacy-an-intervention-to-improvehealth-outcomes.

24. Frisch AL, Camerini L, Diviani N, Schulz PJ. Defining and measuring health literacy: how can we profit from other literacy domains?. Health promotion international. 2012;27(1):117-26.

25. Fransen MP, Van Schaik TM, Twickler TB, Essink-Bot ML. Applicability of internationally available health literacy measures in the Netherlands. J Health Commun. 2011;16(sup3):134-49.

26. Bostock S, Steptoe A. Association between low functional health literacy and mortality in older adults: longitudinal cohort study. Bmj. 2012 Mar 15;344:e1602.

27. Baker DW. The meaning and the measure of health literacy. J gen intern med. 2006;21(8):878-83.

28. SheikhSharafi H, Seyedamini B. Assessment of health literacy and self-care in heart failure patients. Journal of Health Literacy. 2017;1(4):203-19. [Persian]

29. Ilmarinen P, Juboori H, Tuomisto LE, Niemelä O, Sintonen H, Kankaanranta H. Effect of asthma control on general health-related quality of life in patients diagnosed with adult-onset asthma. Scientific reports. 2019;9(1):1-11.

30. Marks GB, Dunn SM, Woolcock AJ. A scale for the measurement of quality of life in adults with asthma. J clin epidemiol. 1992;45(5):461-72.

31. Nassehi A, Abbaszadeh A, Borhani F, Jafari M. Effect of two educational models based on compliance and empowerment on the quality of life of patients with asthma. Journal of Nursing Education. 2013;2(1):1-7. [Persian]

32. Parker RM, Baker DW, Williams MV, Nurss JR. The test of functional health literacy in adults. $J$ gen intern med. 1995;10(10):537-41.

33. Montazeri A, Tavousi M, Rakhshani F, Azin SA, Jahangiri K, Ebadi M, Naderimagham S, Solimanian A, Sarbandi F, Motamedi A, Sistani MM. Health Literacy for Iranian Adults (HELIA): development and psychometric properties. Payesh (Health Monitor). 2014;13(5):58999. [Persian]

34. Saatchi M, Panahi MH, Ashraf Mozafari A, Sahebkar M, Azarpakan A, Baigi V, Holakouie Naieni K. Health literacy and its associated factors: A population-based study, Hormuz Island. Iranian Journal of Epidemiology. 2017;13(2):136-44. [Persian]

35. Panahi R, Osmani F, Sahraei M, Ebrahimi S, Shamsizadeh Nehadghashti M, Javanmardi E. Relationship of Health Literacy and Quality of Life in Adults Residing in Karaj, Iran. J Educ Community Health. 2018;4(4):13-9. [Persian]

36. Parker W. Health Literacy Among Elderly Hispanics and Medication Usage. Thesis, Walden University, Minnesota. 2016.

37. Rosas-Salazar C, Apter AJ, Canino G, Celedón JC. Health literacy and asthma. J Allergy Clin Immunol. 2012;129(4):935-42.

38. Jordan JE, Buchbinder R, Osborne RH. Conceptualising health literacy from the patient perspective. Patient Educ Couns. 2010;79(1):36-42.

39. Soones TN, Lin JL, Wolf MS, O'Conor R, Martynenko M, Wisnivesky JP, Federman AD. Pathways linking health literacy, health beliefs, and cognition to medication adherence in older adults with asthma. J Allergy Clin Immunol. 2017;139(3):804-9.

40. Federman AD, Wolf MS, Sofianou A, O'Conor R, Martynenko M, Halm EA, Leventhal H, Wisnivesky JP. Asthma outcomes are poor among older adults with low health literacy. Journal of Asthma. 2014;51(2):162-7.

41. Mancuso CA, Rincon M. Impact of health literacy on longitudinal asthma outcomes. Journal of general internal medicine. 2006;21(8):813-7.

42. Urbstonaitis R, Deshpande M, Arnoldi J. Asthma and health related quality of life in late midlife adults. Res Social Adm Pharm 2019;15(1):61-9.

43. Chen A, Nowrouzi-Kia B, Usuba K. Health-related quality of life in Canadians with asthma: A case-control study using census data. Respiratory medicine. 2018;140:82-6.

44. Pedram Razi SH, Bassampour SH, Kazemnejad AN. Quality of life in asthmatic patients. Journal of hayat. 2007;13(1):29-34. [Persian] 
45. Shiekhi S, Issazadegan A, Khademi A, Hafeznia M. The comparisons of psychopathology dimensions and quality of life (qol) in people with and without asthma. The Journal of Urmia University of Medical Sciences. 2013;24(7):518-26. [Persian]

46. Couture ÉM, Chouinard MC, Fortin M, Hudon C. The relationship between health literacy and quality of life among frequent users of health care services: a cross-sectional study. Health and quality of life outcomes. 2017;15(1):137:1-6.

47. Zheng M, Jin H, Shi N, Duan C, Wang D, Yu X, Li X. The relationship between health literacy and quality of life: a systematic review and meta-analysis. Health and quality of life outcomes. 2018;16(1):201.

48. Londoño AM, Schulz PJ. Influences of health literacy, judgment skills, and empowerment on asthma self-management practices. Patient Educ Couns. 2015;98(7):908-17.

49. Song S, Lee SM, Jang S, Lee YJ, Kim NH, Sohn HR, Suh DC. Mediation effects of medication information processing and adherence on association between health literacy and quality of life. BMC Health Serv Res. 2017;17(1):661. 